\title{
O papel do farmacêutico em sistemas públicos e universais de saúde: um panorama comparado do Brasil, Canadá e Portugal
}

\author{
The pharmacist's role in public universal health \\ systems: an overview of Brazil, Canada and Portugal
}

\section{El papel del farmacéutico en los sistemas de salud públicos y universales: una comparación visión general de Brasil, Canadá y Portugal}

\author{
Eleonor Minho Conill ${ }^{1}$ \\ Mônica Alves Damasceno²
}

\begin{abstract}
RESUMO: Apesar do reconhecimento da importância da assistência farmacêutica, há dificuldades na integração deste profissional ao sistema de saúde e na atenção primária (APS). O estudo faz uma síntese das normas referentes à assistência farmacêutica no Brasil, com ênfase na inserção do farmacêutico na APS. Estes elementos são comparados com os modelos adotados no Canadá e Portugal, países que possuem sistemas públicos universais orientados por essa política. Foi realizada revisão bibliográfica com as seguintes palavras-chave: "Assistência Farmacêutica", "Atenção Farmacêutica", "Política Nacional de Assistência Farmacêutica" e "Atenção Primária à Saúde" através do Pubmed, Google Acadêmico, sites governamentais, Conselhos profissionais e organismos que constituem fontes para comparações internacionais. O Brasil é o país com o sistema mais generoso do ponto de vista de oferta de medicamentos, além de apresentar grande número de normas que regulam a assistência farmacêutica. Outra singularidade se refere à presença do farmacêutico nas Unidades prestadoras de atenção básica. Com relação ao Canadá observa-se a existência de uma lista limitada de medicamentos autorizados por cada Província e uma proposta de seguro para medicamentos - o Pharmacare. Em Portugal, o governo adota uma lista também limitada para reembolso de medicamentos, que devem ser prescritos pelo nome genérico. Além disso, há um movimento dos farmacêuticos para integração das farmácias ao sistema. Conclui-se mostrando a centralidade dessa discussão para o dilema de ampliar o acesso a medicamentos sem favorecer um consumo desfavorável à saúde e a sustentabilidade dos sistemas.
\end{abstract}

Palavras-chave: Sistema de Saúde. Assistência Farmacêutica. Brasil. Portugal. Canadá.

1 Médica. Doutora em Políticas e programação no desenvolvimento. IEDES/Université de Paris I, Sorbonne. Professora aposentada, Departamento de Saúde Pública, UFSC. eleonorconill@gmail.com

2 Farmacêutica. Especialista em Direito Sanitário. IDISA, Campinas. Farmacêutica na Prefeitura Municipal de Paulínia. monicafcf@hotmail.com 
ABSTRACT: Although the recognition of the matter of pharmaceutical assistance, there are difficulties to integrate this professional to the Health System and to the Primary Health Care - PHC. The study synthesizes the pharmaceutical assistance rules in Brazil, emphasizing the role of the pharmacist in such extent. These elements are compared with adopted models in Canada and Portugal, countries which have public universal systems oriented to PHC. It was performed a bibliographical review with the following keywords: "Pharmaceutical Assistance", "Pharmaceutical Care", "National Policy of Pharmaceutical Assistance" and "Primary Health Care" through Pubmed, Scholar Google, websites of governments and international organizations. Brazil is the country with the most generous system, under the pharmaceutical's offering point of view, and bringing up a large number of rules that regulate the pharmaceutical assistance. Another peculiarity refers to the presence of the pharmacist in the Primary Health Care Units. Canada has a limited list of authorized medicines for each Province and there is a medicine insurance proposal - the Phamacare. In Portugal, the government adopts an also limited list of reimbursed medicines, which shall be prescribed by generic name. Furthermore, there is an action of the pharmacists to integrate the pharmacies to the system. It closes showing the centrality of this discussion to the dilemma of expanding the access of medicines without favoring an excessive consumption unfavorable to health and to the sustainability of the systems.

Keywords: Pharmaceutical Assistance. Pharmaceutical Care. National policy of Pharmaceutical Assistance. Primary Health Care.

RESUMEN: A pesar del reconocimiento de la importancia de la asistencia farmacéutica, hay dificultades en la integración de este profesional al sistema de salud y en la atención primaria. El estudio hace una síntesis de las normas referentes a la asistencia farmacéutica en Brasil, con énfasis en la inserción del farmacéutico en la atención primaria. Estos elementos se comparan con los modelos adoptados en Canadá y Portugal, países con sistemas públicos universales guiadas por esta política. Se realizó una revisión bibliográfica con las siguientes palabras clave: "Asistencia Farmacéutica", "Atención Farmacéutica", "Política Nacional de Asistencia Farmacéutica" y "Atención Primaria a la Salud" a través de Pubmed, Google Académico, sitios gubernamentales, Consejos profesionales y organismos que constituyen fuentes para comparaciones internacionales. Brasil es el país con el sistema más generoso desde el punto de vista de la oferta de medicamentos, además de presentar un gran número de normas que regulan la asistencia farmacéutica. Otra singularidad se refiere a la presencia del farmacéutico en las Unidades prestadoras de atención primaria. Con respecto a Canadá se observa la existencia de una lista limitada de medicamentos autorizados por cada Provincia y una propuesta de seguro para medicamentos-Pharmacare. En Portugal, el gobierno también aprobó una lista limitada para el reembolso de los medicamentos que deben ser prescritos por el nombre genérico. Además, hay un movimiento de los farmacéuticos para la integración de las farmacias al sistema. Se concluye mostrando la centralidad de esa discusión para el dilema de ampliar el acceso a medicamentos sin favorecer un consumo desfavorable a la salud y la sostenibilidad de los sistemas.

Palabras clave: Sistema de Salud. Asistencia Farmacéutica. Brasil. Portugal. Canadá. 


\section{INTRODUÇÃO}

A Organização Mundial da Saúde (OMS) tem produzido uma série de recomendações relacionadas aos medicamentos e ao papel do farmacêutico nos sistemas de saúde, contribuindo para a construção conceitual do campo da atenção farmacêutica. Os documentos divulgados a partir das reuniões de Nova Délhi (1988) e Tóquio (1993) podem ser destacados nesse sentido. Os primeiros ressaltavam a importância do farmacêutico descrevendo suas funções na equipe, especialmente em relação aos seus conhecimentos sobre manejo e propriedades dos medicamentos. O farmacêutico deveria atuar como fonte de informação para a equipe e pacientes, sendo responsável pelo controle de um uso racional e seguro de fármacos. Ou seja, a OMS adota a ideia da farmácia clínica como diretriz para a profissão farmacêutica ${ }^{1}$.

Com a divulgação do trabalho de Hepler e Strand ${ }^{2}$ sobre a atenção farmacêutica houve um novo encontro com a divulgação da Declaração de Tóquio $(1993)^{3}$ que reconhece o farmacêutico como dispensador de atenção, mas ressalta que os benefícios de sua atuação devem ser direcionados ao paciente e à comunidade através de ações que também incluem a promoção e a prevenção. Mais recentemente, a OMS tem insistido na importância de seis pilares para o um desempenho adequado dos sistemas, aos quais denomina de building blocks: prestação de serviços, força de trabalho, informação, acesso aos medicamentos essenciais, vacinas e tecnologias, financiamento e governança com liderança ${ }^{4}$.

A importância de adequar o acesso à assistência farmacêutica tem se tornando central para a sustentabilidade dos sistemas. Um estudo desenvolvido pelo Observatório Iberoamericano de Políticas e Sistemas de Saúde (OIAPSS) ${ }^{5}$ comparou séries temporais de sessenta e cinco indicadores relacionados aos determinantes, condicionantes e ao desempenho dos sistemas de sete países iberoamericanos (Argentina, Brasil, Colômbia, Espanha, Peru, Paraguai, Portugal) durante a primeira década do século XXI. Todos os países apresentaram semelhanças em três aspectos: aumento do sobrepeso, crescimento dos gastos e aumento do desequilíbrio da balança comercial por importação de medicamentos. Este padrão de dependência é mais grave nos latino-americanos, particularmente no caso brasileiro.

Autores ${ }^{6}$ que estudam essa problemática têm apontado a fragilidade da base produtiva do Brasil, apesar do país ocupar a sétima posição no ranking mundial de vendas. A indústria farmacêutica concentra as atividades tecnologicamente mais desenvolvidas nos países centrais, ficando com os demais (a depender do porte de seus mercados) apenas a produção final dos medicamentos ${ }^{7}$. Criase uma dinâmica negativa para os países periféricos: ao mesmo tempo em que expandem o acesso, aumentam o grau de dependência tecnológica com riscos para a sustentabilidade dos sistemas ${ }^{8}$.

Por essa razão, torna-se necessário discutir as questões que se relacionam com a integração tanto do profissional farmacêutico como da assistência farmacêutica ao sistema de saúde. O trabalho contribui neste sentido apresentando um panorama geral da situação em sistemas públicos

ISSN 1982-8829 Tempus, actas de saúde colet, Brasília, 13(2), 53-76, jun, 2019. 
universais com políticas orientadas para a atenção primária de saúde (APS). A primeira parte aprofunda o contexto brasileiro enfocando as principais normas existentes no país, passando a caracterizar na sequência os aspectos relevantes dos sistemas do Canadá e de Portugal.

\section{METODOLOGIA}

Trata-se de um estudo de tipo descritivo com amostra intencional que contemplou os três países já mencionados, tendo como unidade de análise a assistência farmacêutica. Comparar é buscar semelhanças, diferenças ou relações entre fenômenos que podem ser contemporâneos ou não, que ocorram em espaços distintos ou não, para melhor compreendê-los. Estudos descritivos dessa ordem apoiam-se, em geral, no uso de roteiros com os principais componentes dos sistemas (acesso, financiamento, recursos, gestão e prestação), precedidos de uma caracterização do contexto de cada país e, acrescidos das categorias para descrição das ações ou dos serviços que constituem objeto da comparação.

Nesta pesquisa optou-se por uma metodologia que permitisse a descrição integrada das três principais esferas que conformam um sistema de serviços, segundo um modelo proposto por Conill, Fausto e Giovanella ${ }^{9}$ para estudo da APS.

As autoras sugerem que na esfera macrossocial ocorrem decisões políticas acerca dos direitos de acesso, coberturas, financiamento e a macrorregulação (políticas de informação, planejamento, avaliação, desenvolvimento de recursos humanos, científico e tecnológico). Já no nível intermediário, meso ou da gestão, localiza-se um conjunto de atividades de suporte para o desempenho adequado das práticas. Nesse nível importa observar não apenas as decisões, mas principalmente a implementação de mecanismos operativos, tais como, fluxos, suporte tecnológico, formação, comunicação, informação, e regulação profissional. E, por fim, em nível microssocial, onde ocorre o cuidado, as relações interpessoais são importantes, com predomínio dos atributos relacionados ao acesso, à comunicação, informação e à efetividade da atenção.

Ao aplicar esse modelo para assistência farmacêutica, procurou-se identificar a existência no nível macrosocial de normas referentes aos direitos, cobertura, macro regulação de produtos, recursos humanos e política industrial. No intermediário, àquelas que se referem às atividades de gestão, tais como, compras, distribuição, condições de trabalho, entre outras. E no microssocial foi feita uma síntese do que está previsto para a prestação direta de serviços e para a interação entre os usuários, equipe e o profissional farmacêutico (Figura1). 
Figura 1

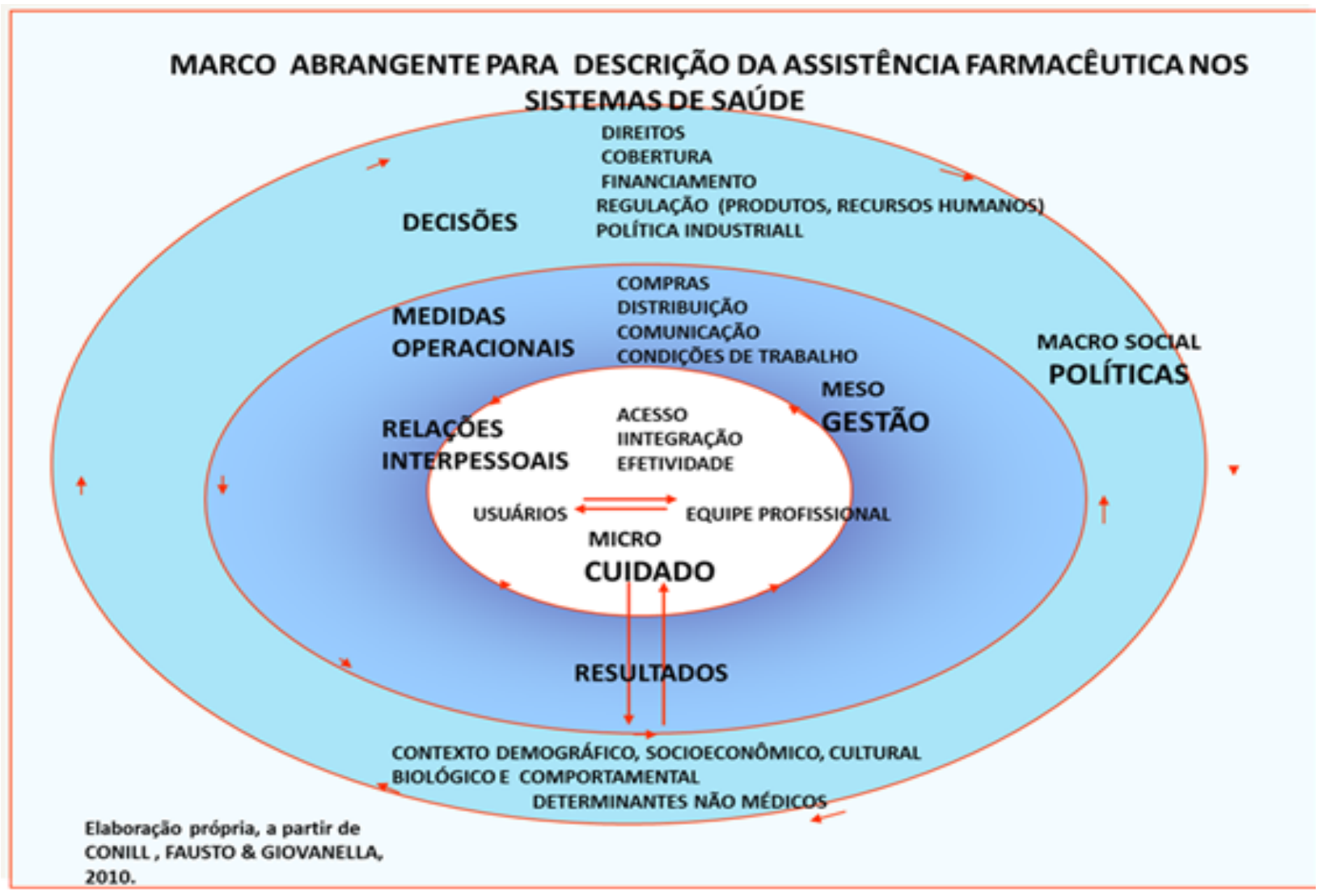

A coleta de dados se deu por meio de pesquisa bibliográfica, a partir das seguintes palavraschave: "Assistência Farmacêutica", "Atenção Farmacêutica", "Política Nacional de Assistência Farmacêutica" e "Atenção Primária à Saúde". As fontes foram o Pubmed, Google Acadêmico para obtenção de literatura cinzenta, sites governamentais, do Conselho Federal de Farmácia e de organismos que são fontes para comparações internacionais, tais como, a Organização Panamericana de Saúde (OPAS), o Instituto Sul-Americano de Governo em Saúde da União de Nações Sul-Americanas (ISAGS), o Observatório Iberoamericano de Políticas e Sistemas de Saúde (OIAPSS), o European Observatory on Health Systems and Policies e a Organization for Economic Co-operation and Development (OECD).

\section{RESULTADOS: A ASSISTÊNCIA FARMACÊUTICA EM SISTEMAS PÚBLICOS UNIVERSAIS}

\section{A assistência farmacêutica no Sistema Único de Saúde do Brasil}

O Brasil é uma república federativa formada por vinte e seis estados e o Distrito Federal, organizados em cinco regiões com importantes diferenças econômicas, culturais e demográficas (norte, nordeste, sul, sudeste e centro-oeste). Conforme os dados mais recentes disponíveis na base da $O E C D^{10}$, o país tem uma população estimada em aproximadamente 201 milhões de habitantes, um Produto Nacional Bruto (PNB) per capita ajustado pela paridade do poder de compra (PPC) da ordem de US\$ 15.065, um Indice de Gini de $0.51^{11}$, sendo que o gasto público com saúde 
corresponde a 3.9 \% do PIB e o gasto privado 5.1\%. As doenças crônico-degenerativas estão entre as principais causas de morbimortalidade, mas o país se caracteriza por apresentar uma elevada mortalidade por homicídios e causas externas. ${ }^{11}$

Criado em $1988^{12}$ e implementado pelas Leis Orgânicas da Saúde $8.080^{13}$ e $8.142^{14}$ de 1990 , o Sistema Único de Saúde (SUS) surgiu inspirado em sistemas nacionais e inserido num modelo de seguridade social. Além da assistência universal, integral e equitativa suas responsabilidades incluem a vigilância (sanitária, epidemiológica, ambiental e de saúde do trabalhador), a formação de recursos humanos, a política de medicamentos, equipamentos, imunobiológicos e outros insumos e, a formulação e execução da política de sangue e derivados. O financiamento advém de fontes fiscais sendo compartilhado entre governo federal, estadual e municipal, com descentralização político-administrativa, mas comando único em cada esfera do governo. Está prevista a colaboração dos entes federados na organização de Redes de Atenção à Saúde (RAS) com os diferentes serviços necessários à integralidade da atenção ${ }^{15}$.

As fontes de financiamento estão organizadas por Fundos em cada instancia de governo e os processos decisórios ocorrem por consenso em Comissões (Tripartite em nível nacional-CIT e Bipartites nas Unidades Federadas). Os serviços de alta complexidade são responsabilidade da União, a média complexidade é garantida por Estados e a atenção básica pelos municipios. Um aspecto positivo na implementação do SUS foi o incremento da atenção básica desde o final da década de 1990. Inicialmente um programa, a Estratégia da Saúde da Família (ESF) passou a ser considerada o principal caminho para reorientação do modelo assistencial. As Equipes de Saúde da Família (EqSF) são constituídas por médico, dentista, pessoal de enfermagem, agentes comunitários, atuam nas Unidades Básicas de Saúde (UBS) e devem ser a porta de entrada do SUS ${ }^{16}$.

Ao serem criados em 1930, os Institutos de Aposentadorias e Pensões incluíam a assistência farmacêutica entre suas responsabilidades, o que pode ser considerada uma das primeiras políticas públicas deste campo. Em 1971 foi criada a Central de Medicamentos (CEME) que funcionou até ser desativada pelo Decreto $N^{0} 2.283$ de 24 de julho de $1997{ }^{17}$. Tinha como característica uma política centralizada de aquisição e de distribuição de medicamentos para a população de baixa renda, incremento à pesquisa químico farmacêutica e à instalação de fábricas de matérias-primas e laboratórios pilotos ${ }^{18}$.

Com a criação do SUS, a Lei Orgânica N ${ }^{0} 8.080 / 90{ }^{13}$ determinou a inclusão de ações de assistência terapêutica integral - inclusive farmacêutica - e a formulação da política de medicamentos (Art. $6^{0}$ ), no campo de atuação do sistema. Este contexto levou a formulação em 1998 da Política Nacional de Medicamentos (PNM) (Portaria GM/MS No 3.916) ${ }^{19}$ tendo como diretrizes: adoção de uma Relação Nacional de Medicamentos Essenciais (RENAME), reorientação da assistência, promoção do uso racional de medicamentos, desenvolvimento científico e tecnológico, promoção da produção, garantia da segurança, eficácia e qualidade dos medicamentos e desenvolvimento de recursos humanos. 
Em 2003, foi criada a Secretaria de Ciência, Tecnologia e Insumos Estratégicos (SCTIE) à qual compete, por intermédio do Departamento de Assistência Farmacêutica e Insumos Estratégicos (DAF), a formulação, implementação e avaliação das Políticas Nacionais de Assistência Farmacêutica e de Medicamentos, incluindo hemoderivados, vacinas, imunobiológicos e outros insumos relacionados ${ }^{20}$.

Em 2004, por meio da Resolução No 338 do Conselho Nacional de Saúde ${ }^{21}$ foi aprovada a Política Nacional de Assistência Farmacêutica (PNAF) que define a assistência farmacêutica como ações para promoção, proteção e recuperação da saúde, tanto individuais como coletivas. O medicamento é considerado um insumo essencial sendo necessário garantir o acesso e seu uso racional. Estas ações abrangem pesquisa, desenvolvimento e produção, bem como, a seleção, programação, aquisição, distribuição, dispensação, garantia de qualidade dos produtos e serviços, acompanhamento e avaliação de sua utilização para melhoria da qualidade de vida da população.

A PNAF integra a política nacional de saúde e seus eixos estratégicos preconizam: acesso e equidade com manutenção de serviços de assistência farmacêutica na rede pública nos diferentes níveis de atenção; qualificação desses serviços com desenvolvimento de recursos humanos, descentralização e definição pactuada de responsabilidades, utilização da RENAME (atualizações periódicas) e uma política de vigilância sanitária para produtos seguros e eficazes.

Também inclui a modernização e ampliação da produção dos Laboratórios Farmacêuticos Oficiais para suprimento do SUS e referências de custo e qualidade da produção de medicamentos, incluindo-se fitoterápicos. Estão previstas ações intersetoriais para uso de plantas medicinais e fitoterápicos com incentivo à produção e a biodiversidade existente no país (políticas de emprego, renda, qualificação de produtores, estímulo para incorporação desta opção terapêutica junto aos profissionais)..

Ações que se referem ao desenvolvimento e inovação do complexo produtivo são igualmente mencionadas através da implementação intersetorial, e em particular com o Ministério da Ciência e Tecnologia, de uma política pública de desenvolvimento científico e tecnológico. Essa política deve envolver os centros de pesquisa e as universidades de modo a atender interesses nacionais e, necessidades do SUS. Inclui mecanismos para a regulação e o monitoramento do mercado de insumos e produtos estratégicos para a saúde.

No que se refere às normas para gestão (nível meso ou intermediário) é possível constatar a referência a um amplo rol de atividades que dizem respeito à organização dos serviços de assistência farmacêutica nos três níveis de atenção no âmbito local e regional. Essas atividades incluem planejamento e avaliação, normatização de procedimentos técnicos e administrativos, estimativas de necessidades com gerenciamento da aquisição e armazenamento, gestão de estoques, manutenção de cadastro dos usuários, unidades e profissionais, desenvolvimento de sistema de informação e comunicação, recursos humanos, pesquisas, material educativo e outras ações que 
assegurem qualidade.

O financiamento é responsabilidade das três esferas de gestão, com os recursos federais sendo repassados até 2017 em seis blocos (atenção básica, média e alta complexidade ambulatorial e hospitalar, vigilância e assistência farmacêutica ${ }^{22}$. O bloco da assistência farmacêutica era composto pelos seguintes componentes: atenção básica e seus programas; parte fixa (per capita a ser complementado por Estados e municípios); parte variável (per capita destinado a programas como hipertensão e diabetes, asma, saúde mental, tabagismo,etc); componente estratégico para tuberculose, hanseníase, malária, leishmaniose, doença de Chagas, antiretrovirais, doenças sexualmente transmissíveis, sangue, hemoderivados e imunobiológicos; medicamentos de dispensação excepcional.

A partir de janeiro de 2018, com a Portaria GM/MS n. 3992/2017, os repasses passaram a ser realizados em apenas duas categorias: custeio e investimento. A medida teve como objetivo agilizar a gestão diminuindo barreiras administrativas, mas foi objeto de críticas devendo ser acompanhada com cautela. ${ }^{23,16}$

Além das normas referidas, é necessário assinalar outras iniciativas importantes na direção de ampliar o acesso ao uso seguro e racional dos medicamentos: a criação da Agência Nacional de Vigilância Sanitária em 1999; a implementação de uma política de genéricos (Lei 9.787 de 1999); o Programa Farmácia Popular do Brasil em 2004 (fornecimento em rede própria a baixo custo e, posteriormente, em farmácias conveniadas), com distribuição gratuita de medicamentos para hipertensão e Diabetes a partir de $2011^{18}$ e, em 2012, o Programa Nacional de Qualificação da Assistência Farmacêutica no âmbito do SUS (Qualifar-SUS) com eixos de trabalho em estrutura, educação, informação e cuidado. ${ }^{24}$

\subsection{A atuação do farmacêutico em âmbito municipal e na Atenção Básica de Saúde (AB)}

As funções do farmacêutico no âmbito da $A B$ se dividem em ações técnico-gerenciais e técnico-assistenciais. As primeiras dão suporte a logística necessária a prescrição e dispensação do medicamento. As técnico-assistenciais estão centradas em garantir a utilização correta do que foi prescrito para obtenção de resultados positivos..$^{25}$

Caracterizam-se como ações técnicogerenciais: a participação no planejamento, organização e coordenação da assistência farmacêutica para regularidade do abastecimento (aquisição, armazenamento, distribuição, rastreamento); elaboração e divulgação, juntamente com outros profissionais, da Relação Municipal de Medicamentos; definição dos medicamentos a serem fracionados e manipulados com acompanhamento de sua produção, elaboração e divulgação de informes, protocolos emateriaisinformativos, incluindo a implementação de Normas eProcedimentos Operacionais Padrão. Também inclui o acompanhamento da utilização de medicamentos com estudos e propostas para melhorá-la, tais como, capacitações e treinamentos, discussões com gestor 
e equipe além da interlocução entre serviços de diferentes níveis de complexidade.

Para as ações técnico-assistenciais está previsto: identificar necessidades e realizar ações de educação voltadas para a comunidade, prescritores e funcionários da farmácia, em conjunto com a equipe da ESF e Núcleo Ampliado de Saúde da Família (NASF); realizar visitas domiciliares e atendimentos individuais quando necessários, participar de planos terapêuticos; garantir dispensação especializada com prevenção de problemas relacionados à medicamentos e conciliação de prescrições visando seu uso racional; realizar gestão de caso junto a outros profissionais para adesão ao tratamento; orientar usuários quanto à obtenção de medicamentos nas três esferas governamentais; promover ações de educação permanente para funcionários das farmácias locais. Abrange igualmente a participação no manejo das condições crônicas, programas estratégicos (tuberculose, hanseníase, DST/AIDS, endemias dentre outras), campanhas, eventos na comunidade, no Conselho Local de Saúde e no Programa de Gerenciamento de Resíduos de Serviços de Saúde. O estímulo à notificação de reações adversas a medicamentos, melhorias da ambiência e humanização nas unidades, incorporação da homeopatia e fitoterapia, recobrem as ações tanto gerenciais quanto assistenciais.

Em 2014, uma publicação do Ministério da Saúde, intitulada Cuidado Farmacêutico na Atenção Básica ${ }^{26}$ fazumasistematizaçãoabrangente do medicamento enquanto insumo essencial especificando em detalhes o escopo das ações a serem realizadas. É interessante mencionar a importância atribuída para atividades de clínica farmacêutica visando o uso racional de medicamentos, diminuição de efeitos adversos e adesão ao tratamento, Também são destacadas ações de conciliação de fármacos que podem ser necessárias nas transições entre os níveis assistenciais (tratamentos prescritos na alta hospitalar que necessitam adequação na $\mathrm{AB}$ ).

Em dezembro de 2007, o Ministério da Saúde propôs o programa "MAIS SAÚDE", por meio do qual estabelecia a criação de 1.500 Núcleos de Apoio à Saúde da Família (NASFs) até $2011^{27}$. Em 2017, com a nova Política Nacional de Atenção Básica (PNAB), os NASFs foram atualizados e passaram a se chamar de Núcleos Ampliados de Saúde da Famíliaa ${ }^{28}$.

O NASF é uma iniciativa que tem aumentado os componentes vinculados às ESF, dentre eles o farmacêutico. De acordo com a Portaria 2448/20112 ${ }^{29}$, que substituiu a anterior, podem ser instituídos dois tipos de NASF, 1 e 2, ambos podendo contar com a participação do farmacêutico. A inserção do farmacêutico é considerada uma iniciativa importante para viabilizar as diretrizes da ESF, da PNM e da PNAF. Publicação do Conselho Federal de Farmácia ${ }^{30}$ sobre as ações do farmacêutico no NASF reiteram as atividades já mencionadas e previstas nessas políticas.

Barberato et $\mathrm{al}^{31}$ realizaram uma revisão sobre o trabalho do farmacêutico na atenção primária no Brasil, selecionando publicações do período 1998-2016 que abordavam experiências, atribuições, potencialidades e desafios. Os estudos analisados foram feitos com profissionais, usuários e gestores. As atividades referidas coincidem com aquelas já mencionadas tanto na área de gestão 
como da assistência (organização e garantia de disponibilidade de medicamentos, uso racional, informações, entre outras). O principal nó crítico diz respeito à inserção do farmacêutico na equipe com dificuldades no reconhecimento e aceitação das intervenções desse profissional.

Um estudo realizado por Goppinger ${ }^{32}$ mostrou que as Unidades de Farmácia Municipais estão localizadas dentro das Unidades Básicas de Saúde (UBS) e ocupam espaços relativamente pequenos. Essa característica foi observada tanto em UBSs antigas quanto nas reformadas ou novas. Nesse pequeno espaço organizam-se áreas de recebimento, armazenamento e dispensação dos medicamentos. Em praticamente todas as Unidades, estão em local externo ou à parte do restante da UBS e a dispensação é realizada através de uma "janela" ou balcão envidraçado. Esta conformação não facilitaria a integração, reforçando o vínculo do serviço farmacêutico com o modelo centrado na consulta médica, no qual a farmácia apenas atende às demandas e pouco participa do cuidado.

\section{Assistência Farmacêutica no Medicare canadense}

O Canadá é uma monarquia parlamentarista, sendo constituído por três territórios na Região Norte e dez províncias nas regiões Ocidental, Central, Oriental e Atlântica. Tem uma população estimada em 35.5 milhões de habitantes, um PNB/per capita (PCC) de US\$ 46.000, um Índice de Gini de 0.31 , sendo que o gasto público com saúde corresponde a $7.3 \%$ do PIB e o privado $3.1 \%{ }^{33}$. O câncer e as doenças cardíacas são as duas principais causas de mortalidade, com um perfil epidemiológico fortemente influenciado pelo envelhecimento populacional. O país apresenta um bom desempenho na prevenção de internações por condições sensíveis à atenção primária, tais como asma e diabetes não controlado, o que sugere cuidados adequados nestes níveis de atenção ${ }^{34}$.

Originalmente, o sistema de saúde estava estruturado com base em um seguro público hospitalar tendo se transformado, com a aprovação do Medical Care Act em 1966, em um seguro de saúde universal conhecido por Medicare. Trata-se de um sistema fortemente descentralizado, tendo base orçamentária fiscal e financiamento compartilhado entre o governo federal e as províncias. Considerando-se a autonomia de gestão pode- se afirmar que o sistema é composto por dez sistemas provinciais e três territoriais com diversos formatos, sempre respeitando os princípios gerais da lei federal: universalização, gestão pública, integralidade, acessibilidade e portabilidade dos direitos no território canadense ${ }^{35}$.

O fornecimento dos serviços se dá através de prestadores privados contratados pelo governo. Os médicos exercem sua prática de forma liberal, atendendo em clínicas particulares e em hospitais, sendo remunerados pelas Agências provinciais que gerenciam o seguro saúde. Os hospitais são em sua maioria (95\%) instituições sem fins lucrativos, administrados por organizações comunitárias, religiosas ou autoridades provinciais. Os seguros privados atuam de forma limitada e complementar, oferecendo serviços não cobertos pelo sistema público, como por exemplo, cirurgias estéticas, hotelaria especial nos hospitais e tratamento odontológico ${ }^{36}$. 
O Québec foi a última província a implantar o seguro de saúde universal, porém ultrapassou os princípios propostos pelo governo federal, destacando-se pelo caráter inovador das ações empreendidas. Essa província apresenta em sua trajetória ações que procuraram redirecionar o foco assistencial hospitalar para a atenção primária, inicialmente com a criação de Centros Locais de Serviços Comunitários (década de 1970) e, posteriormente, com a implementação de Grupos de Medicina de Família ${ }^{35}$.

No Canadá, há uma divisão das funções entre as esferas de governo. É responsabilidade do governo federal as patentes dos medicamentos, a aprovação das drogas que poderão ser prescritas e a regulação da competitividade dos produtos no mercado, interferindo no preço desses produtos. Compete às províncias, por sua vez, garantir o financiamento das medicações ${ }^{37}$.

O sistema garante acesso gratuito às medicações de uso intra-hospitalar, mas não necessariamente aquelas que são utilizadas fora deste ambiente, variando o grau de financiamento público de acordo com as legislações de cada província. Tal análise é realizada por essa esfera de governo, valendo-se de estudos de custo-efetividade das medicações. No entanto, na medida em que o governo federal regula os preços, mas não é responsável pela sua compra, a esfera federal pode comprometer a aquisição de medicações quando não ocorre uma articulação adequada com os governos provinciais. ${ }^{37}$

Convém destacar a declaração da OMS de que é obrigação de todas as nações garantir o acesso equitativo aos medicamentos necessários através de políticas farmacêuticas que funcionam em conjunto com sistemas mais amplos de cobertura de saúde universal. Apesar de ser um país desenvolvido com um sistema deste tipo, o Canadá não garante esse tipo de cobertura. A oferta pública de medicamentos varia com base na idade, na renda ou em necessidades médicas específicas (como câncer, HIV ou fibrose cística). No total, os planos públicos de medicamentos cobrem US \$ 12 bilhões em prescrições, ou $42 \%$ de todos os custos de medicamentos prescritos no país ${ }^{38}$.

Os planos de medicamentos privados, por sua vez, cobrem $36 \%$ dos custos de medicamentos prescritos. Esses planos são voluntários em todas as províncias exceto no Quebec, onde os empregadores são obrigados a fornecer seguros privados de medicamentos para seus funcionários ${ }^{38}$.

Mas mesmo no Québec, província que se caracterizou pela ênfase na atenção primária, não ocorreu a inserção e ou a integração do cuidado farmacêutico nos serviços de porta de entrada do sistema. Os farmacêuticos atuam em estabelecimentos comerciais onde fornecem medicamentos cobertos ou não pelo Medicare.

Os pagamentos diretos dos pacientes canadenses respondem por US \$ 6 bilhões em prescrições, ou $22 \%$ dos custos de todos os medicamentos prescritos. Por essa razão, especialistas defendem a proposta de se estruturar um sistema de cobertura universal de medicações, o Pharmacare, que seria implementado até 2020. Esta política, que se agregaria ao Medicare tem por diretrizes: garantia 
de acesso universal às medicações, solidariedade de seu financiamento e prescrição baseada em evidências de custo-benefício. O Pharmacare propõe a manutenção do financiamento realizado pelas províncias, porém agrega o governo federal nesta responsabilidade, respondendo por $25 \%$ dos custos com as medicações ${ }^{38}$.

\section{A assistência farmacêutica no Sistema Nacional de Saúde (SNS) de Portugal}

Portugal é uma república com regime democrático desde 1974 e inserção na União Europeia em 1986. Tem uma população estimada de 10.450 milhões de habitantes, um PNB/per capita (PPC) da ordem de US\$ 31.200, o Índice de Gini de 0.33, sendo que os gastos públicos com saúde correspondem a $6 \%$ do PIB e os privados $3 \% .{ }^{39}$

As principais causas de mortalidade em ordem decrescente são: doenças do sistema circulatório, tumores malignos, doenças do sistema respiratório, doenças digestivas, causas externas e HIV. O país apresenta um importante envelhecimento populacional com $20 \%$ de habitantes com mais de $65 \operatorname{anos}^{40}$.

O sistema de saúde se caracteriza pela sobreposição de três subsistemas: o Sistema Nacional de Saúde (SNS) instaurado após a revolução de 1974, que é um sistema universal com financiamento fiscal; seguros de saúde associados a categorias profissionais, e seguros de saúde privados voluntários ${ }^{41}$.

O planejamento e a regulação são realizadas pelo Ministério da Saúde enquanto que a gestão é feita por regiões administrativas de saúde, criadas em 1993. As regiões também são responsáveis pela contratação de serviços públicos e privados para a prestação de cuidados. Na teoria estão previstas maiores responsabilidades financeiras, mas estas se limitam à atenção primária visto que os orçamentos hospitalares são alocados a nível ministerial ${ }^{41}$.

Em comparação com a média europeia (24\%), a proporção do financiamento privado é mais elevada em Portugal (35\%), sendo que mais de $80 \%$ disso se aplica a pagamentos de bolso, principalmente atendimento ambulatorial privado, medicamentos, atendimento odontológico e copagamentos no SNS (embora as isenções cubram grande parte da população). Os hospitais públicos são financiados através de orçamentos globais, com um papel cada vez maior do financiamento baseado em atividades por grupos de diagnóstico. O Ministério da Saúde aloca fundos para as regiões com base em uma combinação de despesas históricas e capitação, que pagam pelos cuidados primários e programas específicos de saúde ${ }^{41}$.

Não há no país uma Lista Nacional de Medicamentos Essenciais, mas uma lista positiva de medicamentos reembolsados no setor ambulatorial. Para pacientes internados existe um Formulário Farmacêutico do Hospital Nacional que inclui medicamentos que podem ser usados em hospitais do $\mathrm{SNS}^{40}$. 
As Diretrizes Nacionais de Tratamento Padrão (DTPs) para as doenças mais comuns são produzidas pelo Ministério da Saúde em Portugal. Existem DTPs específicas para cuidados primários, secundários e condições pediátricas, porém o número de estabelecimentos que têm uma cópia dessa norma não é conhecido. Um centro nacional público de informação sobre medicamentos subsidia prescritores, dispensadores e consumidores, tendo sido realizadas campanhas de educação sobre uso racional de medicamentos em 2009 e $2010^{40}$.

Existe uma estratégia nacional escrita para conter resistência antimicrobiana e uma força-tarefa intersetorial para coordenar a promoção do uso adequado de antimicrobianos sendo referido, no entanto, a existência de dificuldades para seu financiamento. Um laboratório nacional de referência é responsável pela coordenação da vigilância epidemiológica da resistência antimicrobiana ${ }^{40}$.

Os prescritores do setor privado não dispensam remédios subvencionados pelo SNS. Existem regulamentos que exigem que os hospitais organizem e desenvolvam Comitês Terapêuticos e de Medicamentos (CTMs). Sabe-se que mais da metade dos hospitais gerais e de referência têm um CTM. A prescrição por nome genérico é obrigatória tanto no setor público e como no privado. Existem disposições legais para reger as práticas dispensadoras do pessoal farmacêutico. O currículo básico inclui informações sobre medicamentos e farmacologia clínica, mas não contempla o conceito da lista nacional de medicamentos essenciais, o uso das diretrizes nacionais de tratamento padrão e a gestão do abastecimento de medicamentos. A educação continuada desses profissionais não é obrigatória. A substituição de equivalentes de genéricos no ponto de dispensação é permitida no setor público e privado. Existe um código de conduta de associação profissional que rege o comportamento profissional dos farmacêuticos ${ }^{40}$.

O processo de compra pública é centralizado sob responsabilidade do Serviços Partilhados do Ministério da Saúde (SPMS), que faz parte do Ministério da Saúde e possui certa autonomia. O procedimento para aquisição é baseado na legislação européia (Diretrizes européias 2004/17 / CE e 2004/18 / CE transpostas para a lei portuguesa através do Decreto-Lei n. ${ }^{\circ}$ 18/2008). Os métodos de licitação no setor público incluem licitações competitivas nacionais e internacionais e compras diretas. Um hospital do SNS pode comprar medicamentos através de contratos que é a principal política de preço, com divulgação pública do processo de licitação (documentação, empresas vencedoras). Não existem disposições legais que deem prioridade a produtos de origem nacional em contratos públicos.

Quanto à distribuição no setor público, o Departamento de Sistema de Abastecimento do governo em Portugal tem um estoque central a nível nacional. Existem guias nacionais sobre boas práticas de distribuição com uma autoridade que emite licenças e acredita as instalações públicas de distribuição ${ }^{40}$. 


\subsection{Atuação Farmacêutica em Portugal}

Em 1999, foi criado o Departamento de Cuidados Farmacêuticos da Associação Nacional das Farmácias (ANF) cujos objetivos eram a criação, divulgação e monitoramento dos programas de cuidados farmacêuticos nas farmácias portuguesas. Em 2001, ocorreu a implementação dos cuidados farmacêuticos através da realização de um ensaio piloto com programas específicos para a diabetes, a hipertensão arterial, a asma e doença pulmonar obstrutiva crônica (DPOC). Nestes casos, a intervenção farmacêutica consistiu no seguimento farmacoterapêutico do doente envolvendo, sempre que necessário, o médico assistente ${ }^{42}$. Os resultados deste ensaio piloto foram avaliados pelo Centro de Estudos e Avaliação em Saúde (CEFAR) tendo sido verificado mudanças positivas no controle dos níveis tensionais e da glicemia pós-prandial, após seis meses do início dos programas ${ }^{43}$. De acordo com outro estudo para avaliar os cuidados farmacêuticos na diabetes, verificou-se que $21 \%$ dos pacientes não controlados integrados ao programa atingiram um controle glicêmico após três meses de seguimento, demonstrando a efetividade deste tipo de serviço ${ }^{43}$.

Cabe também, mencionar o trabalho de outros grupos de investigação que têm procurado estimular a implementação dos cuidados farmacêuticos através de atividades semelhantes, tais como, o Grupo de Investigação em Cuidados Farmacêuticos da Universidade Lusófona (GICUFULHT) e o Grupo de Acompanhamento Farmacoterapêutico de Évora (GAFE) ${ }^{44}$.

Uma revisão da literatura de 2006 refere que Portugal constitui um exemplo de uma abordagem integrada devido ao esforço dos organismos profissionais, sobretudo a ANF e a Ordem dos Farmacêuticos. Aponta o desenvolvimento de um plano e de ferramentas para a gestão de doenças e da terapêutica, criando formas de documentação, softwares e protocolos de intervenção para apoio à esses profissionais ${ }^{45}$.

\section{DISCUSSÃO}

A revisão realizada mostra o caráter particular do modelo assistencial brasileiro ao integrar os serviços de farmácia nas Unidades Básicas de Saúde. Mas são referidas dificuldades para o exercício da atenção farmacêutica neste âmbito e para integração do profissional farmacêutico junto à equipe. Segundo Goppinger ${ }^{32}$, a atividade de orientação aos usuários na farmácia da UBS se tornaria difícil por estar no final do processo de atendimento para onde convergem as mazelas do sistema. Como exemplo, cita prescrições em receituários errados (principalmente de medicamentos de controle especial), falta de medicações e informações nas prescrições, tempo de espera para atendimento médico e na farmácia, dentre outros. O farmacêutico teria uma interação limitada com a equipe por ter seu tempo preenchido com problemas operacionais da gestão dos estoques e atendimento aos usuários, sendo que as ações para reduzir filas tendem a ser valorizadas em detrimento de suas demais atribuições.

A revisão feita por Barberato et $\mathrm{al}^{31}$ confirma a existência de dificuldades apontando o número reduzido de farmacêuticos, a falta de clareza de suas atribuições e as barreiras para integração à 
equipe. Por outro lado, os artigos revisados neste estudo também assinalam aspectos positivos. Destacam que a potencialidade desta proposta foi reforçada através de algumas estratégias recentes, tais como, a presença do farmacêutico no NASF e mudanças na formação profissional com novas Diretrizes Curriculares.

Foi possível identificar a existência de um grande número de normas referentes aos três níveis de ação necessários ao funcionamento de um sistema (das políticas, da gestão e da interação com os usuários). Mas há um distanciamento entre o previsto nessas normas e a realidade dos processos de trabalho. Scherer, Pires \& $\mathrm{Sch}$ artz ${ }^{46}$ tem realizado estudos sobre esse tema na área da saúde utilizando o recurso da démarche ergologique. Segundo esse referencial, trabalhar é gerir a distância entre as normas heterodeterminadas provenientes dos espaços macro (sociais, políticas, econômicas) e dos espaços meso e micro do trabalho (modelos de gestão, protocolos), em função do que cada intervenção exige. No entanto, os contextos e ambientes de trabalho podem favorecer ou dificultar essas gestões.

A complexidade das intervenções frente às demandas na área da saúde vem exigindo que a interdisciplinaridade esteja presente nas práticas de saúde sendo necessário que haja uma recomposição do trabalho em equipe. Mudanças dessa ordem enfrentam dificuldades, pois esbarram nas raízes históricas da divisão técnica do trabalho no desenvolvimento das profissões de saúde.

Além disso, é necessário considerar as dificuldades enfrentadas ao longo da implementação do SUS. Sua arquitetura sugere uma proposta bastante democrática, mas a experiência revela uma gestão fragmentada com alta rotatividade política, amarras legais, administrativas, com uma multiplicidade de instâncias e instituições. Isto traz dificuldades para melhorar e manter estabelecimentos, contratar serviços e construir uma rede integrada, mesmo com as medidas que se sucederam ao longo dos anos, para atenuar essa situação. Além disso, em contradição com o objetivo constitucional de ampliação da cobertura que fundou o SUS, ocorreu um baixo patamar de financiamento público (menor que 50\%), com crescimento de seguros privados para trabalhadores de setores mais dinâmicos da economia ou para a população dos estratos de renda mais altos ${ }^{16}$. A atual conjuntura de recessão e a aprovação da Emenda Constitucional 95/2016 ${ }^{47}$ que congela despesas primárias do Governo Federal por 20 anos pode significar retrocessos nas conquistas que foram obtidas, apesar das adversidades mencionadas. São reconhecidos os avanços importantes da assistência farmacêutica no Brasil que se caracteriza por um amplo modelo de distribuição pública, o que destaca o país em comparações internacionais, principalmente, se realizada com outros países em desenvolvimento ${ }^{18}$.

Portugal apresenta como singularidade um movimento interessante por parte dos profissionais de farmácia visando melhorar a integração com o SNS. Um estudo realizado em $2006^{48}$ comparou a implementação dos cuidados farmacêuticos em treze países europeus (Portugal, Bélgica, Dinamarca, Alemanha, Inglaterra, Islândia, Malta, Escócia, Irlanda do Norte, República da Irlanda, Suécia, cantão alemão da Suíça e País de Gales). A Dinamarca apresentou a menor proporção desses 
cuidados (4\%), sendo a Irlanda o país com maior disseminação desta prática (43\%). Com relação a Portugal, 20\% das farmácias relataram que realizavam a prestação de cuidados farmacêuticos, sendo a informação e a referência de pacientes as atividades mais bem pontuadas. Apesar do aumento dos gastos com medicamentos e dos problemas do uso não racional, esse trabalho revelou que os cuidados farmacêuticos são ainda limitados na União Europeia. Os profissionais raramente estavam, por rotina, envolvidos em atividades centradas no usuário, tais como, a orientação na terapêutica e seu acompanhamento.

Mas a iniciativa identificada em Portugal parece apontar uma tendência de mudança nesse cenário. Também no National Health Service (NHS) na Inglaterra, as farmácias que distribuem medicamentos subvencionados pelo sistema são consideradas parte dos NHS Local Services, com uma política governamental de estímulo a busca do cuidado farmacêutico ${ }^{35}$.

No Canadá, a preocupação com o aumento dos gastos com medicamentos suscitou a proposta de criação de um seguro público complementar com essa finalidade, alternativa que se assemelha ao que foi feito no Medicare americano ${ }^{35}$. O quadro 1 sintetiza os principais elementos da descrição realizada.

Segundo Bermudez, Oliveira \& Luiza ${ }^{18}$ os medicamentos são responsáveis em média por $23.1 \%$ dos gastos mundiais em saúde, mas o maior impacto ocorre nos países de baixa renda. Dados da Pesquisa de Orçamentos Familiares (2008-2009) citados pelos autores mostram que no Brasil eles eram um importante componente da despesa em saúde. Correspondiam à aproximadamente $80 \%$ nas famílias com renda até dois salários mínimos e apenas a $2 \%$ naquelas com vinte e cinco. A proporção dos gastos com medicamentos em relação ao orçamento do Ministério da Saúde dobrou na primeira década do séc.XXI. Um aumento significativo dos gastos com medicamentos de dispensação excepcional e um crescente movimento de judicialização tem sido apontados como preocupantes $^{49}$. Este tema ultrapassou as fronteiras do mundo acadêmico e institucional, sendo objeto de matérias nos meios de comunicação $0^{50}$. O estudo feito pelo OIAPSS ${ }^{5}$ mostrou que o desequilíbrio da balança comercial na importação de produtos farmacêuticos é particularmente grave no caso brasileiro.

No que diz respeito à macroregulação e a gestão para enfrentamento desta problemática foram identificadas um conjunto de medidas no cenário mundial, com destaque para o incentivo ao uso de genéricos, um maior rigor na introdução de novos fármacos nas listas subvencionadas pelos governos e o estímulo às atividades de farmacovigilância ${ }^{35,51}$. 
Quadro 1. Síntese comparada das características da assistência farmacêutica no Brasil, Canadá e Portugal.

\begin{tabular}{|c|c|c|c|}
\hline & Brasil & Canadá & Portugal \\
\hline $\begin{array}{l}\text { Direitos/ } \\
\text { cobertura }\end{array}$ & Universal, integral & $\begin{array}{c}\text { Limitada a lista } \\
\text { de medicamentos } \\
\text { autorizados por cada } \\
\text { província }\end{array}$ & $\begin{array}{l}\text { Limitada a lista } \\
\text { de medicamentos } \\
\text { reembolsados }\end{array}$ \\
\hline Macro-regulação & $\begin{array}{c}\text { Federal, abundância } \\
\text { de normas } \\
\text { RENAME }\end{array}$ & Federal & $\begin{array}{c}\text { Nacional } \\
\text { Lista de medicamentos } \\
\text { reembolsados }\end{array}$ \\
\hline Gestão & $\begin{array}{l}\text { Federal, Estadual e } \\
\text { Municipal }\end{array}$ & Províncias & Nacional e Regional \\
\hline $\begin{array}{c}\text { Papel do } \\
\text { farmacêutico } \\
\text { na prestação de } \\
\text { serviços }\end{array}$ & $\begin{array}{c}\text { Hospitalar } \\
\text { Farmácias na atenção } \\
\text { básica } \\
\text { Farmácias comerciais } \\
\text { pouco integradas }\end{array}$ & $\begin{array}{c}\text { Hospitalar } \\
\text { Farmácias } \\
\text { comerciais } \\
\text { dispensam } \\
\text { medicamentos } \\
\text { subvencionados } \\
\end{array}$ & $\begin{array}{c}\text { Hospitalar } \\
\text { Movimento dos } \\
\text { farmacêuticos para } \\
\text { integração das farmácias }\end{array}$ \\
\hline $\begin{array}{c}\text { Particularidades/ } \\
\text { problemas }\end{array}$ & $\begin{array}{l}\text { Desequilíbrio } \\
\text { da balança, } \\
\text { Judicialização }\end{array}$ & $\begin{array}{c}\text { Proposta de seguro } \\
\text { para medicamentos- } \\
\text { Pharmacare }\end{array}$ & $\begin{array}{c}\text { Obrigatoriedade da } \\
\text { prescrição de genéricos }\end{array}$ \\
\hline
\end{tabular}

Fonte: Elaboração própria.

\section{CONCLUSÃO: A assistência farmacêutica, o modelo assistencial e a sustentabilidade dos} sistemas de saúde

A implementação de sistemas públicos universais visa diminuir desigualdades no acesso, mas este princípio impõe medidas de racionalidade para que os sistemas possam ser sustentáveis. É necessário ter em mente a diferença entre a percepção da qualidade em nível individual e coletivo. Na primeira, a qualidade é percebida como positiva quando obtemos de forma rápida um cuidado resolutivo, já na dimensão coletiva a qualidade deve ser julgada em termos de equidade e gastos. O desempenho dos sistemas de saúde orientados pela atenção primária tem demonstrado sua efetividade nesse sentido. O papel do farmacêutico se tornará cada vez mais necessário para enfrentar os impasses decorrentes da expansão do acesso, dos serviços e das demandas relacionadas com a atual transição demográfica e epidemiológica.

A doença se tornou uma mercadoria altamente geradora de valor e as características dos medicamentos tornam sua regulação particularmente complexa: inelasticidade da demanda por sua importância na preservação da saúde, assimetria de informações, alto grau de concentração dos mercados, entre outros. 
Nos três sistemas analisados identificaram-se alternativas distintas, tanto para incorporação do profissional farmacêutico na atenção básica como para a adequação da assistência farmacêutica. O Brasil é o país com o sistema mais generoso do ponto de vista de oferta de medicamentos, com um grande número de normas que regulam esse campo. Outra singularidade é a presença do farmacêutico nas Unidades prestadoras de atenção básica. Com relação ao Canadá observase uma lista limitada de medicamentos autorizados por cada Província e uma proposta de seguro complementar - o Pharmacare. Em Portugal, o governo adota uma lista também limitada para reembolso de medicamentos, que devem ser prescritos pelo nome genérico. Além disso, há um movimento dos profissionais para integração das farmácias ao sistema. No Brasil, no entanto, exceto por programas nacionais como "Aqui tem farmácia popular", no qual a dispensação de medicações para doenças crônicas é feita gratuitamente em farmácias privadas, e pela fiscalização realizada pela vigilância sanitária, estas se apresentam como serviços paralelos, sem relação com o SUS.

Apesar das diferenças nos contextos, o acompanhamento dessas propostas e modelos, traz informações importantes para o dilema de ampliar o acesso a medicamentos sem favorecer um consumo desfavorável à saúde e a sustentabilidade dos sistemas universais.

\section{Contribuição das autoras:}

As autoras realizaram conjuntamente todas as etapas: entrevistas, análises, estruturação e escrita do artigo.

\section{REFERÊNCIAS}

1. Organización Mundial de la Salud (OMS). El papel del farmacéutico en la atención a la salud: informe de un grupo de consulta de la OMS. Nueva Delhi, India, 13-16 dic, 1988. Genebra: WHO: 1990. 27p.

2. Hepler CD, Strand LM. Opportunities and responsibilities in pharmaceutical care. Am J Hosp Pharm. 1990; 47(3): 533-43.

3. Organización Panamericana de la Salud/Organización Mundial de la Salud (OPAS/OMS). El papel del farmacéutico en la atención a salud: informe de la reunión de la OMS, Tokio, Japon, 31 ago al 3 sep 1993. Buenas Prácticas de Farmácia: Normas de Calidad de los Servicios Farmacéuticos. La Declaración de Tokio. Federación Internacional Farmacéutica. Washington: PAHO: 1995. 31p.

4. Organização Mundial de Saúde (OMS). Monitoring The Building Blocks of Health Systems: A Handbook Of Indicators and Their Measurement Strategies. Genebra: OMS: 2010. 93p.

5. Conill EM, Xavier DR, Piola SF, Silva SF, Barros HS, Báscolo E. Determinantes 


\section{$71 / /$}

sociais, condicionantes e desempenho dos serviços de saúde em países da América Latina, Portugal e Espanha. Ciênc. saúde coletiva [Internet]. 2018 Jul [citado 2019 Maio 28]; 23(7): 2171-2186. Disponível em: http://www.scielo.br/scielo.php?script=sci_arttext\&pid=S1413$81232018000702171 \&$ lng=pt. http://dx.doi.org/10.1590/1413-81232018237.07992018.

6. Gadelha CAG, Maldonado JMSV, Costa LS. O complexo produtivo da saúde e sua relação com o desenvolvimento: um olhar sobre a dinâmica da inovação em saúde. In: Giovanella L et al. Políticas e sistemas de saúde no Brasil, 2a ed. rev. e ampl. Rio de Janeiro: Editora FIOCRUZ: 2012: 209-237.

7. Piola SF. Relatório técnico de análise da matriz de indicadores. Observatório Iberoamericano de Políticas e Sistemas de Saúde (OIAPSS) [Internet]. 2011 [citado 2019 Maio 28]. Disponível em: http://www.oiapss.org/wp-content/uploads/2015/07/Data-03-08-2015Relato\%CC\%81rio-Te\%CC\%81 cnico-de-Ana\%CC\%81lise-da-Matriz-de-Indicadores-doObservato $\% \mathrm{CC} \% 81$ riosugest $\% \mathrm{C} 3 \% \mathrm{~B} 5$ es.pdf.

8. Iñesta A, Oteo LA. La industria farmacêutica y la sostenibilidad de los sistemas de salud desarrollados y America latina. Cien Saud e Colet. 2011; 16(6): 2713-2724.

9. Conill EM, Fausto MCR, Giovanella L. Contribuições da análise comparada para um marco abrangente na avaliação de sistemas orientados pela atenção primária na América Latina. Rev. Bras. Saude Mater. Infant. [Internet]. 2010 Nov [citado 2019 Maio 28]; 10 (Suppl 1): s14-s27. Disponível em: http://www.scielo.br/scielo.php?script=sci_arttext\&pid=S151938292010000500002\&lng=pt. http://dx.doi.org/10.1590/S1519-38292010000500002.

10. Organization for Economic Cooperation and Development (OECD). Countries. Country statistical profile: Brazil [Internet]. 2019 [citado 2019 Maio 23]. Disponível em: https://www. oecd-ilibrary.org/economics/country-statistical-profile-brazil-2019-2_g2g9e77a-en.

11. Observatório Iberoamericano de Políticas e Sistemas de Saúde (OIAPSS). Matriz de Indicadores [Internet]. 2019 [citado 2017 Agosto 19]. Disponível em: http://oiapss.icict.fiocruz. br/resultado.php.

12. Brasil. Constituição da República Federativa do Brasil [Internet]. 1988 [citado 2019 Maio 28]. Disponível em: http://www.planalto.gov.br/ccivil_03/constituicao/constituicaocompilado. htm.

13. Brasil. Lei No 8.080, de 19 de Setembro de 1990. Dispõe sobre as condições para a promoção, proteção e recuperação da saúde, a organização e o funcionamento dos serviços correspondentes e dá outras providências. [Internet]. 1990 [citado 2017 Agosto 19]. Disponível em: http://www.planalto.gov.br/ccivil_03/leis/L8080.htm. 
14. Brasil. Lei No 8.142 de 28 de Dezembro de 1990. Dispõe sobre a participação da comunidade na gestão do Sistema Único de Saúde (SUS) e sobre as transferências intergovernamentais de recursos financeiros na área da saúde e dá outras providências. [Internet]. 1990 [citado 2017 Agosto 19]. Disponível em: http://www.planalto.gov.br/ccivil_03/leis/L8142. htm.

15. Brasil. Ministério da Saúde. Portaria no 4.279 de 30 de dezembro de 2010. Estabelece diretrizes para a organização da Rede de Atenção à Saúde no âmbito do Sistema Único de Saúde (SUS). [Internet]. 2010 [citado 2017 Novembro 15]. Disponível em: http://bvsms.saude.gov.br/ bvs/saudelegis/gm/2010/prt4279_30_12_2010.html.

16. Conill EM. O SUS sem dívida e sem mercado: abrindo o debate. Futuros do Brasil. Idéias para Ação. Centro de Estudos Estratégicos, FIOCRUZ [Internet]. Rio de Janeiro: 2017 [citado 2019 Maio 28]. Disponível em: https://cee.fiocruz.br/sites/default/files/Artigo_Eleonor. pdf.

17. Brasil. Decreto No 2.283 de 24 de julho de 1997. Dispõe sobre a extinção do Instituto Nacional de Alimentação e Nutrição (INAN) e a desativação da Central de Medicamentos (CEME) e dá outras providências. Brasília: 1997.

18. Bermudez JAZ, Oliveira MA, Luiza VL. Assistência Farmacêutica in Giovanella L et AL (Organizadores). Políticas e Sistema de Saúde no Brasil, 2 ed. rev e ampl. Rio de Janeiro: Editora FIOCRUZ: 2012: 657-685.

19. Brasil. Ministério da Saúde. Portaria GM/MS No 3.916 de 30 de outubro de 1998. Aprova a Política Nacional de Assistência Farmacêutica [Internet]. Brasília: 1998 [citado 2019 Maio 23]. Disponível em: http://bvsms.saude.gov.br/bvs/saudelegis/gm/1998/prt3916_30_10_1998.html.

20. Brasil. Decreto No 4.726, de 9 de junho de 2003. Aprova a Estrutura Regimental e o Quadro Demonstrativo dos Cargos em Comissão e das Funções Gratificadas do Ministério da Saúde, e dá outras providências [Internet]. Brasília: 2003 [citado 2019 Junho 02]. Disponível em: http://www.planalto.gov.br/ccivil_03/decreto/2003/d4726.htm.

21. Brasil. Ministério da Saúde. Resolução CNS no 338, de 06 de maio de 2004. Aprova a Política Nacional de Assistência Farmacêutica [Internet]. Brasília: 2004 [citado 2019 Maio 23]. Disponível em: http://bvsms.saude.gov.br/bvs/saudelegis/cns/2004/res0338_06_05_2004.html.

22. Brasil. Ministério da Saúde. Portaria GM/MS nº 204, de 29 de janeiro de 2007. Regulamenta o financiamento e a transferência dos recursos federais para as ações e os serviços de saúde, na forma de blocos de financiamento, com o respectivo monitoramento e controle [Internet]. Brasília: Ministério da Saúde: 2007 [citado 2019 Maio 23]. Disponível em: http:// bvsms.saude.gov.br/bvs/saudelegis/gm/2007/prt0204_29_01_2007_comp.html. 
23. Brasil. Ministério da Saúde. Portal Ministério da Saúde. Novo modelo de financiamento do SUS garante eficiência no uso de recursos [Internet]. 2018 [citado 2018 Janeiro 10]. Disponível em: http://www.saude.gov.br/noticias/agencia-saude/42263-novo-modelo-de-financiamento-dosus-garante-eficiencia-no-uso-de-recursos.

24. Brasil. Ministério da Saúde. Portaria 1.214 de 13 de junho de 2012. Institui o Programa Nacional de Qualificação da Assistência Farmacêutica no âmbito do Sistema Único de Saúde (QUALIFAR-SUS) [Internet]. Brasília: 2012 [citado 2017 Dezembro 02]. Disponível em: http:// bvsms.saude.gov.br/bvs/saudelegis/gm/2012/prt1214_13_06_2012.html.

25. Conselho Regional de Farmácia de Minas Gerais. A importância do farmacêutico no SUS. Suas Competências e Atribuições nas ações de Saúde Pública/Organizador CASP. CRF/MG. $1^{\mathrm{a}}$ Ed. Belo Horizonte: CRF/MG: 2011. 28p.

26. Brasil. Ministério da Saúde. Secretaria de Ciência, Tecnologia e Insumos Estratégicos. Departamento de Assistência Farmacêutica e Insumos estratégicos. Serviços farmacêuticos na atenção básica à saúde [Internet]. Brasília: Ministério da Saúde, 2014 [citado 2019 Maio 22]. Disponível em: http://bvsms.saude.gov.br/bvs/publicacoes/servicos_farmaceuticos_atencao_ basica_saude.pdf.

27. Brasil. Ministério da Saúde. Secretaria de Atenção à Saúde. Departamento de Atenção Básica. Portaria GM 154/2008. Cria os Núcleos de Apoio à Saúde da Família (NASF) [Internet]. Brasília: 2008 [citado 2019 Maio 23]. Disponível em: http://bvsms.saude.gov.br/bvs/saudelegis/ gm/2008/prt0154_24_01_2008.html.

28. Brasil. Ministério da Saúde. Secretaria de Atenção à Saúde. Departamento de Atenção Básica. Política Nacional de Atenção Básica [Internet]. Brasília: 2017 [citado 2019 Maio 23]. Disponível em: http://bvsms.saude.gov.br/bvs/saudelegis/gm/2017/prt2436_22_09_2017.html.

29. Brasil. Portaria GM/MS N 2.488, de 21 de outubro de 2011. Aprova a Política Nacional de Atenção Básica, estabelecendo a revisão de diretrizes e normas para a organização da Atenção Básica, para a Estratégia Saúde da Família (ESF) e o Programa de Agentes Comunitários de Saúde (PACS) [Internet]. Brasília: 2011 [citado 2019 Maio 23]. Disponível em: http://bvsms. saude.gov.br/bvs/saudelegis/gm/2011/prt2488_21_10_2011.html.

30. Conselho Federal de Farmácia. A assistência farmacêutica no SUS/Conselho Federal de Farmácia, Conselho Regional de Farmácia do Paraná; organização Comissão de Saúde Pública do Conselho Federal de Farmácia, Comissão de Assistência Farmacêutica do Serviço Público do CRF-PR [Internet]. Brasília: Conselho Federal de Farmácia: 2009 [citado 2019 Junho 02]. Disponível em: http://www.sbfc.org.br/site/admin/conteudo/pdfs/3174449762.pdf.

31. Barberato LC, Scherer MDA, Lacourt RMC. O farmacêutico na atenção primária no 
Brasil: Uma inserção em construção. Cien Saude Colet [Internet]. 2018 março [citado 2019 Maio 22]. Disponível em: http://www.cienciaesaudecoletiva.com.br/artigos/o-farmaceutico-na-atencaoprimaria-no-brasil-uma-insercao-em-construcao/16679?id=16679.

32. Goppinger KQ. O profissional farmacêutico no âmbito do SUS: um enfoque à assistência farmacêutica. Trabalho de Conclusão do Curso de Especialização em Gestão de Organização Pública em Saúde. Universidade Federal de Santa Maria [Internet]. 2013 [citado 2019 Junho 02]. Disponível em: http://www.paho.org/salud-en-las-americas-2012/index.php?option=com _ docman\&view=download\&category_slug=sna-2012-capitulos-pais-28\&alias $=248$-canada248\&Itemid=125\&lang $=$ en.

33. Organization for Economic Cooperation and Development (OECD). Country statistical profile: Canada 2019 [Internet]. 2019 [citado 2019 Maio 23]. Disponível em: https://www.oecdilibrary.org/economics/country-statistical-profile-canada-2019-2_g2g9e462-en

34. Organização Panamericana da Saúde (OPAS). Saúde das Américas, capítulo Canadá [Internet]. 2012 [citado 2017 Agosto 19]. Disponível em: http://www.paho.org/salud- en-lasamericas-2012/index.php?option $=$ com_docman\&view $=$ download\&category_slug $=$ sna-2012capitulos-pais-28\&alias $=248$-canada-248\&Itemid $=125 \&$ lang $=$ en.

35. Conill EM. Sistemas comparados de saúde. In: Campos GWdS, Minayo MCS, Akerman M, Drumond Júnior M, Carvalho YM. Tratado de Saúde Coletiva. São Paulo: Editora Hucitec: 2012: 563-613.

36. Canadá. O Sistema de Cuidados de Saúde no Canadá. Ottawa: 1999. Worldometers. Worldometers [Internet]. 2017 [citado 2017 Setembro 08]. Disponível em: http://www. worldometers.info/.

37. Anis AH. Pharmaceutical policies in Canada: another example of federal-provincial discord. CMAJ, v. 162, n. 4, 2000 fev: 523-526.

38. Morgan SG, Martin D, Gagnon MA, Mintzes B, Daw JR, Lexchin J. Pharmacare 2020: The future of drug coverage in Canada. Vancouver, Pharmaceutical Policy Research Collaboration, University of British Columbia. 2015. 20p.

39. Organization for Economic Cooperation and Development (OECD), "Country statistical profile: Portugal 2019/1", in Country statistical profiles: Key tables from OECD, OECD Publishing. Paris: 2019 [citado 2019 Maio 23]. Disponível em: https://doi.org/10.1787/csp-prttable-2019-1-en.

40. Portugal. Ministry of Health in collaboration with the World Health Organization. Portugal Pharmaceutical Country Profile [Internet]. 2011 [citado 2017 Novembro 
30]. Disponível em: http://www.who.int/medicines/areas/coordination/Portugal_ PSCPNarrativeQuestionnaire_27052011.pdf.

41. Simões J, Augusto GF, Fronteira I, Hernández-Quevedo C. Portugal: Health system review. Health Systems in Transition. 2017: 1-184.

42. Associação Nacional de Farmácias. Contributos em saúde para os diabéticos. Avaliação do Programa de Cuidados Farmacêuticos: Diabéticos. Farmácia Observatório. 2006.

43. Costa S, Madeira A, Santos M, Santos C, Matias L, Helling D. Pharmacy-based disease management in Portugal: preliminary results of a pilot intervention. Working Paper, International Pharmaceutical Federation. 2003.

44. Martins S, Costa FA, Caramona M. Implementação de cuidados farmacêuticos em Portugal, seis anos depois. Rev Port Farmacoter. 2015 [citado 2019 Junho 01];5(4): 4-12. Disponível em: http://revista.farmacoterapia.pt/index.php/rpf/article/view/38.

45. Van Mil JF, Schulz M. A Review of Pharmaceutical Care in Community Pharmacy in Europe [Internet]. Health Highlights. 2006 [citado 2019 Maio 23]: 155-168. Disponível em: https://pdfs.semanticscholar.org/5a85/b26b2bf2a55d19011cdbfdbb4aad40fdad9b.pdf

46. Scherer MDA, Pires D, Schwartz Y. Trabalho coletivo: um desafio para a gestão em saúde. Rev. Saúde Pública [Internet]. 2009 Aug [citado 2019 Maio 30]; 43(4): 721725. Disponível em: http://www.scielo.br/scielo.php?script=sci_arttext\&pid=S003489102009000400020\&lng=en. http://dx.doi.org/10.1590/S0034-89102009000400020.

47. Brasil. Emenda Constitucional n. 95, de 2016. Altera o Ato das Disposições Constitucionais Transitórias para instituir o Novo Regime Fiscal, e dá outras providências. [citado 2019 Maio 26]. Disponível em: http://www2.camara.leg.br/legin/fed/emecon/2016/ emendaconstitucional-95-15-dezembro-2016-784029-publicacaooriginal-151558-pl.html.

48. Hughes CM, Hawwa AF, Scullin C, Anderson C, Bernsten CB, Björnsdóttir I et al. Provision of pharmaceutical care by community pharmacists: a comparison across Europe. Pharm World Sci. 32(4):472-487. 2010 Ago [citado 2019 Junho 01]. Disponível em: https://www. ncbi.nlm.nih.gov/pubmed/20458539.

49. Pepe, VLE et al. A judicialização da saúde e os novos desafios da gestão da assistência farmacêutica. Ciênc. saúde coletiva [Internet], v. 15, n. 5. Rio de Janeiro: 2010 Ago [citado 2017 Dezembro 12]: 2405-2414. Disponível em: http://www.scielo.br/scielo.php?script=sci_ arttext\&pid $=\mathrm{S} 1413-81232010000500015 \& \operatorname{lng}=\mathrm{en} \& \mathrm{nrm}=\mathrm{iso}$.

50. Marasciulo M. Todos por um? Revista Galileu [Internet]. 2017 março 06 [citado 2019 
Junho 02]. Disponível em: https://revistagalileu.globo.com/Revista/noticia/2017/03/todos-porum.html.

51. Organização Panamericana da Saúde. "Boas práticas de farmacovigilância para as Américas" [Internet]. Washington: OPAS: 2011 [citado em 2019 Maio 30]. 76p. Disponível em: https://apps.who.int/medicinedocs/documents/s18625pt/s18625pt.pdf.

Artigo apresentado em: abril 2019 Artigo aprovado em: junho 2019

Conflito de interesses: as autoras declaram não haver conflito de interesses Suporte financeiro: não 